\title{
Die Theorie lebt in der Praxis. Ein Interview mit Ernst Stadlober
}

\author{
Ernst Stadlober \\ TU Graz
}

\author{
Herwig Friedl \\ TU Graz
}

\author{
Matthias Templ \\ TU Wien \& Statistics Austria
}

\begin{abstract}
Das Interview mit Ernst Stadlober wurde von Herwig Friedl und Matthias Templ am 18.12.2015 geführt. Es zeichnet ein Bild des beruflichen Werdeganges von Ernst Stadlober, von seinen Anfängen wo er mit fix gesetztem seed auf deterministischem Wege über die Random Number Generation zu seiner sehr breiten Ausrichtung der Statistik fand. Viele erfolgreich angewandte Forschungsprojekte mit Partnern aus Verwaltung, Industrie und Wirtschaft bezeugen ebenso seine Erfolgsgeschichte als auch die beispiellose intensive Betreuung von Studenten an der TU Graz. Man kann zurecht behaupten, dass Ernst Stadlober ein breites Methodenspektrum aus dem Gebiet der Statistik beherrscht und es trotzdem schaffte in viele Spezialgebiete auch tiefer vorzudringen.
\end{abstract}

Ernst Stadlobers berufliche Heimat war und ist das Statistikinstitut der TU Graz, das er seit 1998 auch leitet. Dazwischen war er auf Forschungsaufenthalten an der Stanford University/USA und der TH Darmstadt und hatte eine Lehrstuhlvertretung an der Universität Kiel. Bis heute hat er 12 Dissertationen und mehr als 90 Diplom-/Masterarbeiten betreut. Zum Repertoire seiner Lehre zählt die (Angewandte) Statistik, Zeitreihenanalyse, Stochastische Modellierung und Simulation, Versuchsplanung und einiges mehr. Zusätzlich blickt er heute auf über eine Reihe von 100 Vorträgen sowie auf etwa 80 Publikationen aus dem Bereich der Biostatistik, Computerstatistik und Angewandten Statistik zurück.

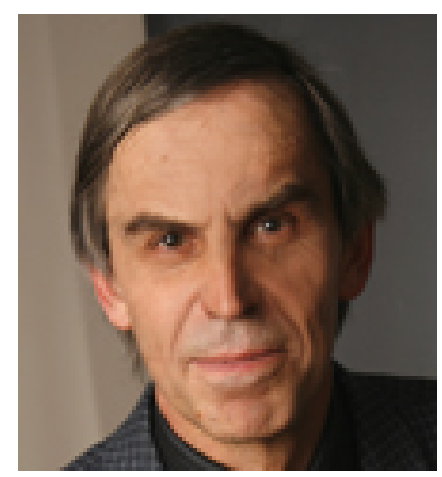

Keywords: Interview, Statistik, Statistikstudium, Projekte.

Matthias Templ: Danke für deine Zusage uns ein Interview zu geben. Nach einer Reihe von sehr interessanten Interviews im Austrian Journal of Statistics, setzen wir die Interviewreihe mit dir fort. Vielleicht sollten wir von deinen Anfängen in der Statistik zuerst sprechen. Am Statistikinstitut der TU Graz hast du bei Ulrich Dieter dissertiert. Meines Wissens war er eine große Persönlichkeit.

Ernst Stadlober: Ja, das war ein sehr umtriebiger, vielseitiger Mathematiker. Der kommt an sich aus der Zahlentheorie und hat eben damals, wie es so üblich war, in der Mathematik an der TU Graz diese Stelle für Mathematische Statistik angenommen. Das 
war das Normale, dass ein Mathematiker so einen Bereich übernommen hat. Prof. Dieter hat dann auch die Vorlesungen Wahrscheinlichkeitstheorie und Statistik gehalten und die natürlich eher aus mathematischer Sicht durchgeführt. Er selbst hat nie selber Statistik betrieben in dem Sinn. Ich bin durch ihn in den Forschungsbereich der Zufallszahlenerzeugung reingekommen. In der Lehre hat er damals am Anfang auch die Optimierungstheorie abgedeckt, weil zu der Zeit haben wir an der TU Graz noch keine Professur dafür gehabt. Mein Einstieg war nicht die Statistik sondern die Optimierung. Da hab' ich dann auch meine Diplomarbeit geschrieben, im Bereich der Optimierung. Das war eine Untersuchung über das so genannte quadratische Zuordnungsproblem, ein Vergleich von Computerheuristiken. Da war schon der Ansatz, wo man sagt: man hat die Kombination zwischen der theoretischen Analyse von Methoden und den praktischen Vergleichen von Methoden - auch am Computer.

Matthias Templ: Und das bereits in den frühen 80er Jahren, mit Veröffentlichungen von dir mit computergestütztem Inhalt und computerorientierten Methoden.

Ernst Stadlober: Ja, wie gesagt, der Einstieg war dann stark computerorientiert. Ich habe die Assistentenstelle 1976 bekommen, knapp nach dem Abschluss meines Diploms. Dann ist zu dieser Zeit auch Rudi Dutter nach Graz gekommen, als Assistent und der hat einen starken Computerbezug mitgebracht. Er hat damals die ersten Vorlesungen in Computerstatistik bei uns angeboten. Also mit SPSS und BMDP zum Beispiel hat er das durchgeführt. Das war dann für mich quasi der Einstieg in die ersten Anwendungen mit realen Datenbeispielen. Dadurch ist man langsam reingekommen in diese Datenlandschaft: wie kann man die Methoden, die man theoretisch gelernt hat, umsetzen, wo sind sozusagen auch die Hemmschwellen. Das war also ein ganz guter Anfang in dieser Problematik. In der Lehre habe ich damals schon relativ viel übernehmen müssen, Übungen und auch eigene Vorlesungen. Obwohl ich das Doktorat noch gar nicht hatte, habe ich zum Beispiel Vorlesungen wie Maßtheorie, Wahrscheinlichkeitstheorie, Warteschlangentheorie oder Stochastische Prozesse abgehalten, neben der Übungsbetreuung und Betreuung von Projekten. Wie gesagt, der Einstieg in die Rechnergeschichte war so, dass wir im Studium Fortran gelernt haben und ich habe in weiterer Folge sehr viel in Fortran programmiert.

Herwig Friedl: Ich kann mich sogar daran erinnern, als Rudi Dutter mit APL gekommen ist und wir dann gemeinsame Seminare gemacht haben, das war dann vielleicht ein paar Jahre später.

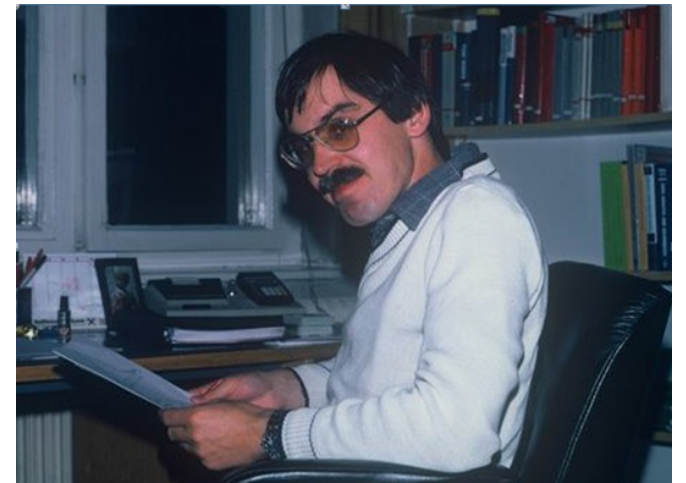

Foto 1981: Assistent an TU Graz

Ernst Stadlober: Ja das APL war damals sehr interessant und spannend. Das Problem in APL war halt die Tatsache, dass die Tastatur anders zu benutzen war. Wenn du „mal“ drücken wolltest beim APL, dann war das Malzeichen ganz woanders zu finden als in Fortran. Und ich kann mich erinnern, ich habe ein Jahr parallel gearbeitet, einmal mit Fortran, einmal mit APL. Dann habe ich APL schließlich aufgegeben, weil das hin und her mit unterschiedlichen Tastaturen war nicht mehr zu packen. Das war das große Hindernis für APL, obwohl es von der Sprache her sehr gelungen war, es war schon vektororientiert und matrixorientiert. Die Matrixinversion war ein Befehl. In Fortran hast du das alles explizit ausprogrammieren müssen mit den Schleifen und so weiter. [lacht $]$

Herwig Friedl: Hast du eigentlich deine Algorithmen in Fortran implementiert, oder auch noch in anderen Sprachen? 
Ernst Stadlober: In Fortran, ja. In APL auch - und in Assembler. Wir haben ja damals auch diese Zufallszahlen untersucht - Prof. Dieter hat sich mit gleichverteilten beschäftigt und Prof. Ahrens, sein Koautor, mit den nicht-gleichverteilten. Da waren die zwei

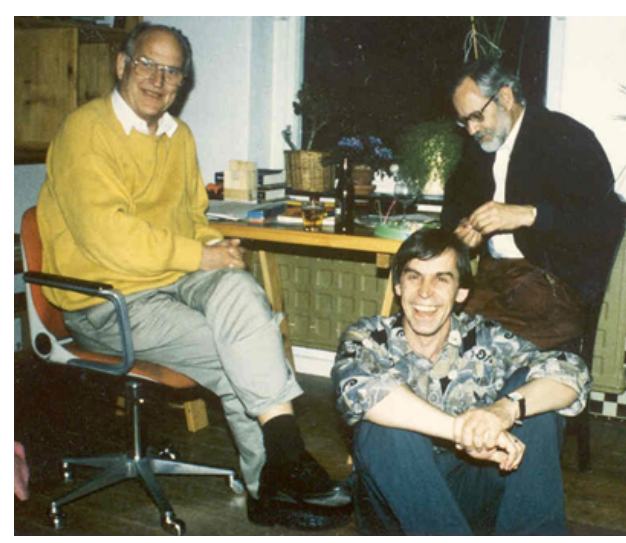

Foto 1993: mit Ahrens und Dieter ja Gurus auf dem Gebiet: Ahrens-Dieter war so eine Trademark in der Scientific Community. Ich habe dann versucht Algorithmen zu finden für bestimmte Verteilungen, und die haben wir dann in Fortran programmiert, aber den Generator für die gleichverteilten Zufallszahlen zum Beispiel, den haben wir immer in Assembler programmiert, weil der war die Basis von allen anderen Algorithmen. Der hat dann möglichst schnell und effizient sein müssen, damals auf einem UNIVAC Großrechner. Wir haben zur damaligen Zeit natürlich alle Tricks ausgenutzt in Richtung effizienter Mul-

tiplikation und Speicherung, um möglichst rechenzeitsparend und auch speichersparend zu arbeiten. Also das war ja eine ganz andere Zeit mit wenig Speicherplatz und langsamen Rechnern. Die Optimierung war immer in der Richtung, dass du Methoden findest, die diese beiden Aspekte gut genug abdecken konnten.

Herwig Friedl: Aber seitdem ich dich kenne hast du immer mehr oder weniger relativ angewandt gearbeitet und bei der Anwendung natürlich auch relativ computergestützt.

Ernst Stadlober: Ja, das auf alle Fälle.

Herwig Friedl: So irgendwie die neue Schiene "Rudi Dutter/Ernst Stadlober" die Dieter überhaupt nicht vertreten hat. Dieter war reiner Theoretiker. Der hat mit einem Blatt Papier und einem Kugelschreiber alles gemacht und bei dir hat man dann gelernt, wie die Sache in der Praxis tatsächlich angegangen werden könnte.

Ernst Stadlober: Ja eben, wobei der Dieter jetzt nicht der Freak war am Computer, hat er trotzdem die Offenheit gehabt dafür. Er hat ja mit Jo Ahrens zusammen gearbeitet, Jo Ahrens war der Computermensch, und Uli Dieter hat eher die theoretischen Sachen entwickelt. Den Vorteil dieser beiden Teile hat er durchaus gesehen und er hat uns nie irgendeinen Hemmschuh in den Weg gelegt und gesagt, ok, der macht jetzt zu viel Anwendung oder arbeitet viel mit Computer, sondern im Gegenteil. Er war zum Beispiel der erste, der das TeX nach Graz gebracht hat zum Beispiel, oder das Maple. Wo damals die Leute im Rechenzentrum gesagt haben: „Was ist denn das?“, so auf die Art. Kannst du dich noch erinnern [zu Friedl], der Johann Theurl war damals der Chef, der war eine recht dominierende Persönlichkeit und hat solche Sachen immer abgeblockt. Wenn der Dieter mit irgendeinem Computerband aus Deutschland gekommen ist, wo interessante neue Programme drauf waren, hat er zunächst abgeblockt. Und wie gesagt, der Dieter hat dann durchaus den Riecher gehabt für neue Entwicklungen und hat uns vieles in dieser Richtung ermöglicht.

Matthias Templ: Ich denke du hattest nie Angst vor Neuem. Ich habe ein bisschen recherchiert, in welchen Bereichen der Statistik und Themengebieten du aktiv warst. Das überspannt Bereiche wie Chemometrie, Biometrie, Versuchspläne, Zeitreihenanalyse und Prognose, Stochastik und Portfolio-Optimierung. Überdies hast du Diplomarbeiten in den verschiedensten Gebieten betreut, z.B. in Themen wie Mischmodelle, Resamplingverfahren, Anwendungen der Linguistik und eben, wie du schon erwähnt hast, Random Number Generators. Wegen dieser sehr breiten Ausrichtung komme ich zum Schluss, dass du sehr offen für neue Themen sein musst. 
Ernst Stadlober: Ja, das Spannende ist, wenn man jetzt als Mathematiker draufblickt auf diese Dinge. Gott sei Dank kommen wir ja aus der Mathematik und wir haben die Strukturen der Methoden gelernt, von der Mathematik her. Dann habe ich eine Anwendung und sehe, eigentlich ist das immer das gleiche Schema für mich als Mathematiker: ich habe das abstrakte Modell, ich muss nur den Übergang schaffen vom Abstrakten zur konkreten Anwendung. Dann ist es vollkommen egal, ob ich mit einem linguistischen Problem arbeite, mit einem Problem aus der Prozessindustrie, mit einem Problem aus der Medizin, ich habe sozusagen immer mein gleiches abstraktes Konstrukt. Das Problem ist also: „Wie schaffe ich den Link dazwischen?“. Das heißt, wie kann ich mit dem, der mit einem Problem zu mir kommt, erst einmal die gleiche Sprache finden. Ich weiß, am Anfang haben wir ja herausfinden müssen - da war der Herwig ja auch immer wieder eingespannt im medizinischen Bereich - was die Leute überhaupt wollen. Also, dass man überhaupt fragt: „Ok, was willst du jetzt überhaupt?“. Das waren oft sehr diffuse Vorstellungen und sehr diffuse Aussagen. Die haben wir zuerst einmal ein bisschen strukturieren und auf den Punkt bringen müssen, das war der erste Schritt. Und dann verstehen, aha, jetzt haben wir es soweit, jetzt können wir schauen, wie passt das zusammen mit irgendeinem statistischen Modell. Also wie kann ich die Fragestellung übertragen - die Merkmale und so weiter. Wenn man diesen Schritt einmal gelernt hat, und versucht, den Partner zu verstehen, dann wird im Prinzip immer der gleiche Mechanismus angewendet, natürlich mit unterschiedlichen Ausprägungen. Also ich sehe das eher so als einheitliches Konstrukt, dass ich sage, egal mit welchem Problem du heute kommst, ich kann das in mein Konzept allgemeiner einbringen. Und das ist das Spannende an unserer Statistik, an der sogenannten Angewandten Statistik, die wirklich angewandt ist, dass du durch die Verbindung zum Abstrakten irrsinnig viel erreichen und machen kannst.

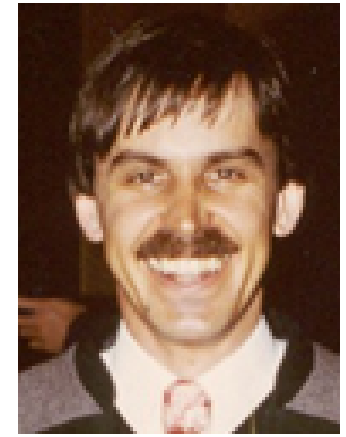

Foto 1983: Promotion

Matthias Templ: Dann gibt es aber heutzutage wahrscheinlich zu wenige Angewandte Statistiker, weil sich immer mehr Wissenschaftler auf einen Bereich spezialisieren. Dort sind sie dann natürlich Spezialisten, aber nicht mehr offen für andere Sachen.

Ernst Stadlober: Wie gesagt, das ist natürlich ein Grundproblem, weil man natürlich jetzt an der Uni primär, wenn man sich habilitieren will, fokussiert sein muss auf so einen engen Bereich. Ich habe mich damals auf meinen Forschungsbereich, die Zufallszahlenerzeugung konzentriert. Da habe ich mich auf Algorithmen gestürzt und neue Verfahren ausgearbeitet und entwickelt. Die Methoden nicht nur entwickelt, sondern auch in entsprechende Packages verpackt, die zugänglich waren. Das Ende war, darauf bin ich heute noch stolz, das Softwarepackage CRAND oder WinRand, das war eine Implementierung von Zufallszahlengeneratoren für über 30 Verteilungen, das in $C$ geschrieben worden ist. Das wurde dann als offene Plattform von uns angeboten. Das war vielleicht ein bisschen wie - von der Größe natürlich überhaupt nicht vergleichbar mit $R$ - aber so ein ganz kleiner Beitrag, wo ich sage, da ist etwas, was man der Öffentlichkeit zugänglich macht, etwas Aktuelles. Das ist es tatsächlich noch immer, Teile davon sind implementiert worden in anderen Packages. Es ist auch in $R$ ein bisschen was von uns drinnen und es gibt immer noch Methoden, die ich vor mehr als 20 Jahren entwickelt habe, die heute nach wie vor am Stand der Technik sind. Also da sieht man, wenn man etwas intensiv betreibt und ernsthaft versucht, es effizient zu machen, dass dies durchaus Bestand haben kann - auch in unserer Zeit, wo es sehr kurzlebig zugeht im Bereich der Softwareentwicklung und so. Aber gewisse Grundprinzipien ändern sich ja trotzdem nicht.

Aber wie gesagt, das was ich jetzt als Vorteil sehe in unserem Bereich ist, dass wir Mathematik studiert haben und ausgehend von der Mathematik haben wir die grundlegende Methodik sauber gelernt und das ist ein Fundament, und wenn Interesse besteht 
auch raus zu gehen, das muss ich mich halt trauen als Mathematiker. Dabei verlasse ich den sicheren Boden von „Definition, Satz und Beweis“, und beschäftige mich mit sogenannten schmutzigen Daten, wo nichts mehr exakt stimmt, von der Theorie her, auf das muss ich mich einlassen. Den Mut haben, auch wenn man am Anfang überhaupt nicht weiß, ob irgendetwas funktioniert, einfach reinzugehen und das auszuprobieren. Das haben wir auch erst lernen müssen. Bei den ersten angewandten Projekten habe ich selber irrsinnig viel Bauchweh gehabt, ob das überhaupt funktioniert und das war die Hemmung des Mathematikers. Weil da habe ich gesagt, jetzt verlasse ich einen sicheren Boden und ich weiß überhaupt nicht was mich da vielleicht erwartet. Scheitere

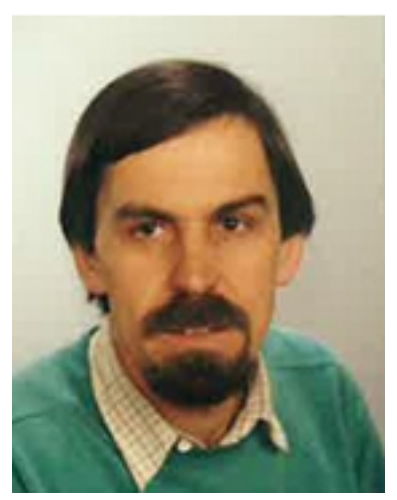

Foto 1988: vor Habilitation ich überhaupt mit meinen Methoden? Und natürlich haben wir dann Schritt für Schritt kleine Erfolge gehabt in der Anwendung und haben gesehen, dass die Modelle ganz gut funktionieren und dass wir das umsetzen können. Und dann wirst du natürlich immer frecher und bekommst immer mehr Selbstbewusstsein, weil du sagst irgendetwas kommt immer heraus im Endeffekt - das ist unser Anspruch, wenn irgendjemand kommt und am Anfang sagt „Ich weiß nicht so recht, ob da was drinnen ist.“, aber ich habe die Erfahrung gemacht „Irgendwas schaffen wir schon, was nicht ganz sinnlos ist.". Und mit dem arbeite ich heute, quasi mit dem Vertrauen das man durch die jahrelange Erfahrung bekommen hat.

Matthias Templ: Das hat sich also insofern weiter entwickelt, dass du irre viele Projekte mit der Wirtschaft und anderen Instituten erfolgreich durchgeführt hast.

Ernst Stadlober: Ja, das ist dadurch entstanden. Am Anfang, wie gesagt, habe ich mich im Bereich der Zufallszahlen habilitiert und habe dann eben, als Dozent die normale Lehrverpflichtung für die Mathematiker gehabt. Dann sind diese angewandten Projekte, so ein paar kleine, gekommen. Das erste war zum Beispiel, da war der Herwig Friedl ja als Student noch dabei, diese Geschichte mit den Lungenfunktionsdaten. Damals hat in der Steiermark Prof. Karl Harnoncourt, ein Bruder von unserem Dirigenten Nikolaus Harnoncourt, das recht groß aufgezogen. AKL hat das geheißen, das Kürzel. Da ist ein Bus etabliert gewesen, der ist in der ganzen Steiermark herumgefahren und hat einfach die Leute zusammen getrommelt, um diesen Test zu machen. Das heißt, das waren natürlich keine selektierten Daten, natürlich nicht so, wie man sich das heute vorstellt bei einer wissenschaftlich sauberen Studie. Das war die sogenannte Normalbevölkerung einfach zusammen gefischt. Die Daten haben wir dann bekommen und das waren, glaube ich, 15000 oder so etwas - erstaunlich viel für die damalige Zeit.

Herwig Friedl: Das war ein Big-Data-Problem.

Ernst Stadlober: Ja und wir sind damals an die Grenzen vom UNIVAC Rechner und dem Programmpaket BMDP gekommen. Wir haben wirklich versucht die Daten zu modellieren. Das waren zwar nur multiple Regressionsmodelle, aber immerhin. Für die damalige Zeit ein irrsinniger Rechenaufwand und da kann der Herwig auch gewisse Schmankerl erzählen, was da alles drinnen war, in diesen Daten bei der Selektion - Identifikation der Leute, zum Beispiel.

Oder ein und derselbe Mensch hat einmal „Joseph Maier“ geheißen und einmal „Maier Joseph“ und einmal „Dr. Maier Josef“. Da haben wir dann, kannst du dich noch erinnern, solche Selektionen gehabt von Listen von Leuten wo wir erkannt haben, der war fünf mal so drinnen und fünf mal anders. Weil die Sozialversicherungsnummer vielleicht auch nicht dabei war. Also es war gar nicht so einfach.

Herwig Friedl: Aber es war eine gewaltige Datensituation jedenfalls. Ich bin mir nicht so sicher, ob du mit 15000 da richtig liegst, ich hab so irgendwie sogar 80000 im Kopf. 
Ernst Stadlober: Im Endeffekt haben wir daraus Österreichische Normwerte entwickelt, für die Mediziner, eingebaut in ihre Apparaturen. Für die Berechnung haben wir die Daten dann natürlich vorher selektiert. Dafür waren immer noch auswertbare Daten in der Größenordnung von über 10000 Probanden vorhanden. Der Vorteil war, dass wir eine europa- oder sogar weltweit einzigartige Bandbreite bzgl. des Alters gehabt haben: Untersuchungen von sechsjährigen Kindern bis 85-jährigen Senioren. Das heißt, die Leute, die mit 85 Jahren noch zum Bus gehen konnten, die waren noch dabei. Es war in dem Sinn natürlich nicht mehr die Normalbevölkerung dieser Altersgruppe, sondern es waren nur die Gesunden drinnen, die extrem Gesunden. Aber immerhin haben wir dann Modelle entwickeln können für diese große Altersbandbreite, was es zur damaligen Zeit sonst noch gar nicht gegegeben hatte.

Herwig Friedl: Kannst du dich noch an diesen Hilfeschrei erinnern, den du bekommen hast, als bei einem Patienten das Modell angewandt worden ist und eine negative geschätzte Vitalkapazität resultiert hat.

Ernst Stadlober: Ja, das Schmankerl muss ich erzählen. Da ruft mich der Arzt aus der Lungenabteilung des LKH an, ganz vorwurfsvoll, dass unser Modell falsch ist: bei einem Probanden kommen negative Werte heraus. Ich habe mir gedacht: „Das gibt es ja gar nicht.". Weil wir hatten das Modell damals evaluiert bezüglich aller möglichen Bereiche, da waren wir immer sehr gewissenhaft und genau und haben jedes Mal, wenn wir irgendetwas aus der Hand gegeben haben, exakt dokumentiert, wofür das Modell gilt; Alter von-bis, Größe von-bis, Geschlecht - immer genau angegeben. Dann frage ich da weiter und sag”: „Was war denn das für ein Patient?“. Dann hat sich herausgestellt, dass das ein Liliputaner war. In unserem Modell ist natürlich die Körpergröße sehr wesentlich eingegangen - das ist klar. Und der Liliputaner war $1.20 \mathrm{~m}$ und für den war natürlich alles falsch. Das Modell war für die Größen von $1.60 \mathrm{~m}$ weg bei den Männern oder so, zulässig. Der Arzt hat das aber extrapoliert hinunter zur Körpergröße des Liliputaners und ist dann natürlich zu negativen Modellwerten gekommen. Daraufhin habe ich den Arzt gefragt: „Haben Sie überhaupt einmal nachgesehen, wofür das Modell eigentlich gilt? Sehen Sie dort irgendeinen Hinweis, dass das auch für Körpergröße 1.20 m gilt?". Ja, dann hat er schön langsam eingesehen, dass das nicht unser Fehler war, sondern er in der Anwendung auch aufpassen muss. Ähnliche Geschichten haben wir oft erlebt mit Anwendern, die uns vorgeworfen haben, dass wir etwas falsch gemacht haben. Aber meistens haben wir das dann ausräumen können, da irgendetwas in der Anwendung nicht so passierte, wie das eigentlich vorgesehen war. Also wir haben generell immer versucht, das, bevor wir etwas aus der Hand gegeben haben, immer für uns zu evaluieren und abzuchecken, dass da nichts schief gehen kann - also nichts grob schief gehen kann.

Ja, aber es war damals eine wertvolle Erfahrung, diese Lungenfunktionen, weil da haben wir das erste Mal, wie der Herwig richtig gesagt hat, mit sogenannten Big-Data gearbeitet. Wir haben also die Möglichkeit gehabt uns durch diese Datenflut zu wühlen und zu selektieren. Das heißt, wir haben eigentlich ein wenig selber geübt und trainiert - wir waren ja auch am Anfang. Heute würde ich das natürlich ganz anders machen, das ist ja klar mit der Erfahrung. Aber immerhin, wir haben eine sehr gute Case-Study gehabt aus der Praxis. Und ein anderes Erlebnis ist auch ganz lustig. Wir haben Untersuchungen gehabt von bestimmten Gruppen wie Feuerwehrmänner zum Beispiel. Aichfeld Murau war damals ein großes Thema in den 80er Jahren, wie die Draken kommen sollten in die Steiermark - die Vorgänger vom Eurofighter. Da hat es in der Steiermark die sogenannte Draken-Studie gegeben, vom Landeshauptmann beauftragt, und da sollten bestimmte Fachleute eben untersuchen, ob die Region Aichfeld vielleicht schon so stark belastet ist, dass zusätzliche Düsenjäger quasi keinen Sinn machen. Da sind wir beauftragt worden, zu vergleichen, ob es Unterschiede gibt in den Lungenfunktionswerten von Personen im Aichfeld und von Personen der Region Murau, aufgrund der Umweltsituation. Da war im Prinzip kein Unterschied nachweisbar. Da hatten wir auch Daten von 
Feuerwehrleuten, daraus haben wir für unsere Studenten einen Datensatz ausgewählt für die Lehre, der heißt aimu-Datensatz. Dieser geistert eben seit Ende der 80er Jahre herum und ist ein recht einfacher Datensatz mit 79 Datenpunkten, wo aber von jedem Feuerwehrmann bestimmte Merkmale drinnen sind. Das heißt du kannst einfache Modelle erklären, du kannst die Verteilungen der Merkmale untersuchen, da hast du stetige Variablen, du hast Gruppierungsvariablen und der Datensatz bringt für uns selber in der Lehre immer wieder neue Überraschungen, weil man die Daten immer wieder aus neuen Blickwinkeln anschaut. Und das ist mittlerweile für uns ein legendärer Datensatz. Wenn wir aimu erwähnen, lachen wir schon und sagen: „Das ist unser aimu-Datensatz, den haben wir schon so lieb gewonnen. Den geben wir nicht mehr aus der Hand.". Und den Studenten ist er auch schon sehr vertraut.

Herwig Friedl: Gekommen bist du ja, wie du schon gesagt hast, von der Zufallszahlengeneratorebene. Dann hast du dich, daran kann ich mich noch gut erinnern, beworben als Nachfolger um die Stelle von Sepp Gölles. Das war für mich damals ziemlich interessant, da ich mir nicht unbedingt vorstellen konnte, dass jemand von der random numbers Seite kommend, in die Angewandte Statistik so ohne irgendetwas hinein schlittert. Du hast die Stelle bekommen, und dann hast du richtig losgelegt mit Anwendungen und Projekten, hast an das Institut eine gewaltige Anzahl von größeren, kleineren, andauernden Projekten mit Firmen, auch mit dem Land Steiermark oder mit der Stadt Graz, geholt und jetzt mehr oder weniger dieses Institut, zumindest diese Seite vom Institut aufgebaut, hier im Land Steiermark eine extrem gut anerkannte, perfekt positionierte Gruppe von Leuten etabliert, die immer wieder in der Lage sind, derartige Studien durchzuführen.

Und das was mir jetzt am Schluss sehr gut gefallen hat, war die Zusammenarbeit mit dem Umweltamt, also dein tolles Engagement in Richtung PM1O oder dergleichen. Am Anfang glaube ich war dies eher eine sehr lokale Problematik wo du da mitgespielt hast - Graz betreffend. Das ist in der Zwischenzeit aber schon viel umfassender geworden. Ihr habt auch zusammengearbeitet mit den Südtirolern, Bozen und so weiter. Kannst du uns da ganz kurz so einen Sketch von dieser Linie erzählen?

Ernst Stadlober: Naja das war so, 1997 habe ich diese Professur, die Nachfolge vom Sepp Gölles, bekommen, und das war natürlich Angewandte Statistik. Da habe ich mir gesagt, ok, es ist klar, jetzt habe ich diese Stelle „Angewandte Statistik“, ich hatte auch als Vorbild Josef Gölles, der ja wirklich einer der Pioniere da war in Graz, der die Statistik nach außen getragen hat in unterschiedliche Bereiche. Er hat auch im Rechenzentrum eine kleine Gruppe gehabt und dann im Joanneum Research, und das war für mich ein Vorbild, dass ich mir sagte, schau, so etwas könnte ich weiter treiben in der Art: ich habe eine gute akademische Basis, habe auch gute Studenten aus der Mathematik zur Verfügung und ich versuche die Studenten schön langsam in diese Richtung zu bringen, jene die interessiert sind für angewandte Projekte. Und wie gesagt, dadurch, dass wir vorher diese Lungenfunktionsgeschichten schon sehr intensiv betrieben haben, bin ich schon selber ein bisschen auf den Geschmack gekommen, dass ich mir dachte, das dürfte auch allgemein interessant werden für andere Themen. Der Anfang war bei uns die medizinische Statistik, von der Anwendung eben, über die Lungenfunktionsschiene. Und diese Umweltproblematik ist dann gekommen, weil ich Kontakte gehabt habe zum Umweltamt und da hatte Graz ein Problem als man angefangen hat diese PM10-Werte zu messen, und die EU-Grenzwerte, die neu eingeführt wurden, nicht einhalten konnte. Dann hat man gleich erkannt: „Graz hat ein Problem, was machen wir?“. Daraufhin haben wir uns zusammengesetzt; das Umweltamt und ich mit Partnern aus der TU Graz haben dann gemeinsam ein EU-Projekt beantragt, zusammen mit Klagenfurt und Südtirol, Bozen, wo wir versucht haben, diese PM10-Problematik zu bearbeiten. Da waren einige Fachleute dabei aus anderen Bereichen, die technische Untersuchungen und Messungen gemacht haben. Oder wir haben auch eine Firma aus Bayern gehabt, die ein neues Messsystem ausprobiert hat. Unsere Aufgabe war es statistisch zu untersuchen, wie es mit der Ver- 
teilung von PM10-Konzentrationen aussieht. Haben wir eine Möglichkeit Prognosen zu rechnen für PM10 Tagesmittelwerte, damit man vielleicht, wenn die Politik auf irgendein System einsteigt, über die Prognosen Hinweise geben kann, wann gewisse Maßnahmen zu setzen sind. Das war eben der Ausgangspunkt, dieses EU-Projekt, wo ich gemerkt habe, dass man als Statistiker mit ganz unterschiedlichen Partnern zusammen arbeiten kann. Als Quintessenz des Projekts haben wir Modelle entwickelt für die Prognose von Tagesmittelwerten, sowohl für Bozen und Klagenfurt, als auch für Graz. Das einzige, was seitdem noch laufend umgesetzt wird sind die Prognosen in der Wintersaison in Graz, weil wir für Graz die Daten online im steirischen Messystem haben und das Grazer Umweltamt so offen ist und uns jedes Jahr damit beauftragt. Für die anderen zwei Städte haben wir zwar die Modelle entwickelt, aber das hat nicht gelebt, weil sich dort niemand mehr darum gekümmert hat. Das ist jetzt so ein Projekt, das läuft mittlerweile seit 2003 glaube ich. Ich habe dann auch Kontakte zu Kollegen in Brünn gehabt und mit denen zusammen, die haben auch ähnliche Modelle für Brünn entwickelt, wurde dann vor kurzem eine Arbeit geschrieben mit einem Modellvergleich, wo wir gezeigt haben, dass wir beides recht gut einbetten können in ein gemeinsames System. Im Prinzip sind das einfache GLMs oder multiple Regressionsmodelle. Das Modell selber ist da nicht so das Entscheidende, sondern das Entscheidende ist: „Was soll ich in das Modell reinbringen?“. Das ist da die Hauptarbeit gewesen. Welche Messgrößen beschreiben mir jetzt zum Beispiel die Meteorologie am besten. Und welche Merkmale bekomme ich auch als Messungen und was habe ich dann sonst noch zur Verfügung.

Das heißt die Hauptarbeit ist da nicht die Methodikarbeit in der Statistik, sondern das Vorfeld, dass ich mir überlegen muss von der Sache her, welche Merkmale habe ich zur Verfügung, welche kann ich zuverlässig messen und welche bringen dann für mein Modell auch Information. Das habe ich auch bei den anderen Projekten immer wieder erkannt: das Wichtigste ist das Vorfeld, dass ich einmal abchecke, was liegt vor und wie kann ich die relevanten Merkmale herausfinden - welche Merkmale soll ich beobachten, damit ich zu einem plausiblen Modell komme mit einer Aussagekraft für die Anwendung.

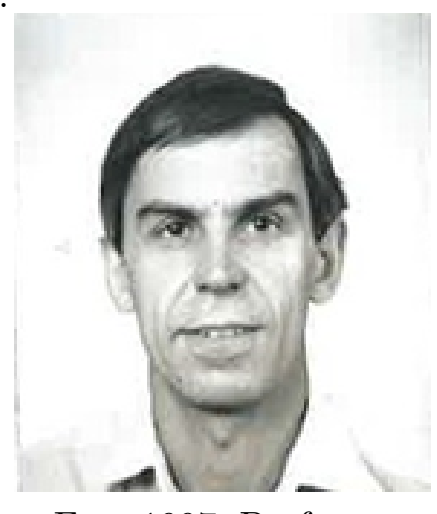

Foto 1997: Professor

Herwig Friedl: Gerade die verschiedenen methodischen Aspekte, die du bei deinen Projekten immer angewandt hast, haben als Quintessenz dann auch eine Modifikation der Lehre hier vor Ort gehabt. Ich denke nur, eine Vorlesung wie Stochastische Simulation hat es am Anfang, als ich studiert habe, nicht gegeben. Eine Vorlesung Angewandte Statistik mit den Inhalten, wie du sie jetzt machst, Design of Experiments, Time Series, das hat es alles mehr oder weniger vor 30 Jahren nicht gegeben. Also haben ja auch diese praktischen Aspekte irgendwie dahingehend einen Einfluss gehabt. Wie siehst du das?

Ernst Stadlober: Das ist wirklich eine sehr gute und spannende Frage, weil ich bin der Ansicht, dass derjenige, der eine Vorlesung durchführt, selbst am meisten davon profitiert. Ich lerne durch eine Vorlesung. Und ich habe immer den Ansatz gehabt, so wie der Herwig gesagt hat, ok, jetzt haben wir bestimmte Themen, wir brauchen gewisse Methoden - wie lernen wir die selber am besten? Ich muss die Methoden und deren Anwendungen ja selber beherrschen. Das Beste ist, wenn ich eine Vorlesung mache auf dem Gebiet, dann lerne ich auch die Details sauber. Ich muss ja tiefer gehen, ich muss den Studenten das beibringen und gleichzeitig lerne ich den Stoff. Sozusagen: ich bin dann der, der Hauptprofiteur ist! Das war typisch bei der Zeitreihenvorlesung zum Beispiel, die hat es vorher nie gegeben, da war niemand dafür da und es war aber der Bedarf da von Studierenden aus anderen Fachrichtungen, Zeitreihen zu lernen. Dann habe ich mir gedacht, mache ich halt die Vorlesung Zeitreihenanalyse, da muss ich mich jetzt einmal hineinknien und das gut verstehen von der Methodik. Ich habe das generell so gemacht mit den Vorlesungen, ich habe immer versucht die mathematische Basis ganz 
sauber zu machen, aber sofort auch mit Anwendungen verbunden. Das heißt, ich habe in jeder Vorlesung natürlich den Computeraspekt drinnen - das heißt, es gibt bei mir keine Statistikvorlesung, wo nicht praktische Übungen sind mit $R$, wo ich selbst auch die Beispiele mit dem $R$ rechne. Die Studenten haben dann kleine Projekte als Übungsaufgaben. Das heißt diese Kombination ist für mich etwas ganz Wertvolles, auch in der Vorlesung: zuerst die Grundmethodik und gleich, wenn die Leute die Grundmethodik können, sofort: „Wie schaut die Umsetzung aus?“. Und wie gesagt, wir haben ja auch nicht nur bei den Vorlesungen diesen Aspekt, dass wir selbst etwas lernen wollen, sondern wir haben bewusst auch unsere Seminare gemeinsam immer so ausgewählt, dass wir uns sagen, jetzt haben wir ein Thema, da gibt es jetzt neue Methoden in dem und dem Bereich, da wissen wir noch zu wenig - machen wir ein Seminar. Das heißt, der Herwig und ich haben jahrelang jedes Semester ein Seminar gehalten, wo wir immer auch wir als Seminarleiter - gelernt und vorgetragen haben. Wir haben bewusst solche Themen gewählt, da wissen wir noch nicht so gut Bescheid, gehen wir in den Bereich hinein - zusammen mit den Studenten. Das heißt, das war für mich der Sinn von Seminaren, dass man nicht irgendein totes Thema gibt, damit die Studenten beschäftigt sind, sondern wir haben immer versucht auch das mit einem Lernprozess für uns zu verknüpfen. Den Blickwinkel haben wir eben gehabt und deswegen haben wir so viele Anwendungen in breiten Bereichen. Ja gut, das ist jetzt verknüpft damit, dass wir uns selber weiter gebildet haben, gemeinsam, sehr breit in der Statistik. Das war nicht nur ich, sondern der Herwig hat ja auch mitgemacht, das heißt, wir haben beide jetzt wirklich ein sehr vielfältiges, breites Portfolio an Methoden, die wir kennen. Ich habe also Seminarordner und elektronische Unterlagen, wo Beispiele ausgearbeitet sind von jedem Bereich und mir ist es oft schon passiert, dass irgendjemand kommt, der sagt, ich habe ein Problem in dem Bereich, dann schnappe ich den entsprechenden Ordner und sage, da hast du den Ordner, da drinnen sind genau die Grundlagen für das, was du brauchst. Da brauchst du kein Buch, da haben wir ausgearbeitete Beispiele von Studenten, die super präsentiert wurden. Darauf haben wir auch immer Wert gelegt: das Seminar war bei uns einerseits lernen für alle und andererseits, die Studenten sollten ja nicht nur den Inhalt lernen, sondern auch die Präsentation beherrschen. Das heißt, wir haben auch sehr viel Wert darauf gelegt, dass die Studenten die Präsentation schriftlich sauber ausarbeiten und dann vortragen. Das was sie programmiert hatten war offenzulegen. Das alles war für uns ein Anliegen, weil heute sollen die Absolventen ja auch diese Softskills können. Und das haben wir versucht mit Inhalt zu füllen. Es war für mich nie ein Anliegen, Studenten in einen Rhetorik-Kurs zu schicken. Da diskutieren sie irgendein Thema und lernen präsentieren, wie man den Computer einschaltet und wie man das vorführt. Wie gesagt, bei uns muss die Präsentation verknüpft sein mit Inhalt. Dann lernt man das für sein Fach. Wie muss ich jetzt mein Fach rüberbringen in der Präsentation und da bin ich nicht unbescheiden, da braucht man nicht unbescheiden sein, weil das hat wirklich gut funktioniert. Wir sehen immer wieder wie toll die Studierenden dann bei der Präsentation der Masterarbeit oder Dissertation agieren. Wir haben jetzt kaum mehr irgendwelche Präsentationen, wo ich sage, die sind nicht brauchbar. Da haben die Studenten auch bei uns ein sehr hohes Niveau erreicht, eben durch diese Schulung im Seminar.

Herwig Friedl: Jetzt wirst du uns ja unglücklicherweise in absehbarer Zeit leider abhanden kommen und diese vier Wände mit privaten Wänden zurück tauschen. Ich möchte zumindest von dir jetzt, vielleicht auch aus rein persönlichem Interesse, ein bisschen darüber etwas hören, wie du die Rolle der Statistik, die Ausbildung in der Statistik, die Zukunft der Projekte hier an der TU Graz siehst.

Ernst Stadlober: Naja, die Zukunft hängt natürlich stark davon ab von welchen Personen das weiter betrieben werden kann. Einer der Mitstreiter ist ja der Herwig, Gott sei Dank, der bleibt ja doch noch einige Jahre tätig und meine Hoffnung ist, dass eben 
meine Nachfolge durchaus auch aus dem Holz geschnitzt ist, dass sie nicht nur im stillen Kämmerlein sitzt und die eigenen Forschungsinteressen verfolgt, sondern auch so ein bisschen ein Typ ist, der vielseitig ist und sich auch nicht scheut da reinzugehen in die Anwendung. Was wir liefern können ist ein gut ausgebautes Netzwerk, womit man am Beginn Unterstützung anbieten kann. Da habe ich sicher kein Problem, dass ich in der Übergangszeit dann mithelfe, wenn noch eine gewisse Scheu besteht Kontakte aufzunehmen. Es wäre schade, weil das Netzwerk ist ja schon vorher vom Sepp Gölles gelegt worden, und ich habe das was der Herr Gölles angefangen hat im Wesentlichen weiter betrieben - natürlich in meiner, in unserer, anderen persönlichen Art, aber ich würde schon sagen, ich habe auch schon in gewisser Weise auf ein bestimmtes Netzwerk aufgebaut. Ich habe es dann halt mit meinen Möglichkeiten ausgefüllt und weiter ausgebaut. Ich hoffe, dass meine Nachfolge das auch nutzt. Es gibt gute Voraussetzungen von verschiedenen Seiten, sie kann das mit ihren neuen Ideen füllen und das vielleicht weiter treiben in Richtung einer Verknüpfung zwischen der guten mathematischen Ausbildung und der Anwendung in Projekten. Und wie gesagt, wir haben immer wieder gute Studenten, die Studierendenbasis ist bei uns sehr gut, die sind immer interessiert an Projekten und ich habe da meistens sehr gute Erfahrungen gemacht mit Studierenden, die in Firmen gearbeitet haben. Die Mitarbeiter in Firmen sind dann immer sehr überrascht von der hohen Qualität unserer Studenten. Ich habe schon einige Studentinnen gehabt, die in Firmen hineingegangen sind, wo keine einzige Frau gearbeitet hat, zum Beispiel bei Magna-Steyr in Graz. Diese Diplomandin hat eine Versuchsplanung gemacht für Klebeprozesse, wo sie den eigenen Versuchsaufbau selber zusammengebaut hat auf einem großen Tisch. Die konnte sich problemlos dort behaupten. Die Mitarbeiter dort waren sehr glücklich, dass endlich auch einmal eine Frau dabei ist, die ein anderes Leben in die Bude bringt. Und wie gesagt, die hat das super gemacht.

Zweites Beispiel, vor kurzem bei der AT\&S in Hinterberg. Da wurde jetzt auch eine Masterarbeit fertig gestellt. Bei einem Termin mit dem erweiterten Vorstand haben die nur so geschwärmt von dieser Studentin: „Die müssen wir behalten, weil die ist so gut und die passt so gut rein in unser Team.". Mittlerweile arbeitet sie auch dort, aber wie gesagt, das ist für mich auch so, dass man dadurch auch gewisse Hürden abbaut, indem man die Studentinnen einfach integriert. So wie bei anderen Problematiken, wenn man etwas nicht kennt, lehnt man einmal ab, aber wie gesagt, da hat es bei uns immer eher positive Erfahrungen gegeben. Frauen bewähren sich jetzt im technischen Bereich. Wir haben vor Kurzem so ein Brainstorming gemacht, was haben wir für eine Geschlechterverteilung bei den Studierenden? Unsere 50 abgeschlossenen Master/Diplomarbeiten in den letzten 10 Jahren wurden von 25 Frauen und 25 Männern geschrieben. Wir erreichen also eine exakte Geschlechterparität. Also das ist bei uns ganz normal ohne viel Zusatzaufwand passiert. Wir haben das irgendwie einfach so genommen wie es ist und wir haben viele gute Studenten ausgewählt, und da waren halt auch genug gute Frauen dabei. Ich kann jetzt nicht sagen, dass ich mit den Studentinnen prinzipiell andere Erfahrungen gemacht habe, als mit den Studenten. Das war ziemlich ausgewogen: in jeder Gruppe gab es Gute und weniger Gute.

Matthias Templ: Ich möchte nur ganz kurz hier anknüpfen und habe eine letzte Frage. Also ich nehme dich auch kurz vor der Emeritierung wahnsinnig aktiv wahr.

Ernst Stadlober: Pensionierung heißt das bei mir! [lacht]

Matthias Templ: Und ich erlebe auch, dass ihr mit Euren Studenten ein familiäres Verhältnis habt.

Herwig Friedl: Ab und zu tanzt er sogar mit ihnen! [lacht]

Matthias Templ: Aber wirst du uns in der Statistik auch weiter erhalten bleiben? 
Ernst Stadlober: Also, wie gesagt, meine Pläne in nächster Zeit sind so, dass ich in der Übergangsphase noch im Pflichtlehrprogramm mitmachen werde. Ich arbeite bereits an kleineren Projekten mit Partnern, die noch über meine Pensionierung hinaus laufen. Wir haben ja auch Kontakte zu unseren Kompetenzzentren, zum Beispiel gibt es das ViF (das virtuelle Fahrzeug) die ganz interessante Problematiken haben, da sitzen auch Diplomanden und Absolventen von uns. Da ist es nach wie vor gut, wenn man einfach mit der Expertise, die man hat als erfahrener Statistiker, dabei ist. Mein Rat wird ja auch offensichtlich noch gewünscht. Ich dränge mich nicht auf, aber anscheinend ist es doch noch so, dass meine Expertise nachgefragt wird. Wie gesagt, ich bin natürlich mit Leib und Seele Statistiker und das werde ich bis zu meinem Lebensende bleiben, aber ich habe auch Gott sei Dank eine Familie, in der ich gut aufgehoben bin, mit einer großen Kinderschar und auch Enkel, das heißt, ich versuche halt beides irgendwie unter einen Hut zu bringen - bis jetzt ist das halbwegs gelungen - und wenn die Gesundheit mitspielt, das weiß man ja nie, werde ich sicher noch einige Zeit mich da einbringen, wenn es gewünscht ist. Das ist keine Frage.

Was wir jetzt nicht konkret erwähnt haben ist, dass wir wahnsinnig viele Diplomanden haben. Also, wir haben überproportional viele im Vergleich zur Mathematik. Da haben wir gerade gestern noch mit dem Vizerektor ein Gespräch gehabt, dass wir da auch extrem stark die ganze Studienrichtung tragen, OR und Statistik, und zusätzlich noch die Finanzmathematik. Ich habe ja auch so eine kleine Präsentation zusammen gestellt, eine Übersicht von den Projekten und Diplomarbeiten, wo auch Zahlen drinnen stehen. Was wir auch an Dissertationen und so betreut haben insgesamt, am Institut. Ich bin ja nicht alleine, aber der Herwig und ich haben in der Statistik gemeinsam sehr viel gemacht - beide haben wir jetzt sehr viel betreut, oder betreuen immer noch. Also, wie gesagt, das ist für mich jetzt schon ein Zeichen, dass es schade wäre, wenn durch die Nachfolgegeschichte irgendetwas schief geht. Wir haben auch einen guten Stand innerhalb der TU Graz, weil ich mit Ingenieuren immer viel zusammen gemacht habe, z.B. Dissertationen betreut. Das hat natürlich auch die Wirkung, dass wir innerhalb der TU Graz auch eine gewisse Anerkennung erfahren. Die schätzen unseren Bereich durchaus. Wir waren natürlich auch meistens bereit, als Ansprechpartner da zu sein. Dazu muss man halt auch bereit sein - es ist immer ein Geben und Nehmen.

Matthias Templ: Dann möchte ich mich herzlich für das Gespräch bedanken.

Herwig Friedl: Genug des Guten.

Ernst Stadlober: Ich danke auch. Genug des Guten.

\author{
Affiliation: \\ Ernst Stadlober \\ Institute of Statistics \\ Graz University of Technology \\ Kopernikusgasse 24/III \\ A-8010 Graz, Austria \\ Email: e.stadlober@tugraz.at
}


Herwig Friedl

Institute of Statistics

Graz University of Technology

Kopernikusgasse 24/III

A-8010 Graz, Austria

Email: HFriedl@TUGraz . at

Matthias Templ

Vienna University of Technology \&

Statistik Austria

A-1040 Vienna, Austria

E-mail: matthias.templ@gmail.com

\section{Austrian Journal of Statistics}

published by the Austrian Society of Statistics

Volume 45

March 2016 http://www .ajs.or.at/

http://www.osg.or.at/

Submitted: 2015-12-18

Accepted: 2016-01-26 